\title{
Development Based on Unity3D Virtual Campus Roaming System
}

\author{
Yao $\mathrm{Hu}^{1,2^{*}}$, Jingwen $\mathrm{Li}^{1,2}$,Xiaoqiang Han ${ }^{1,2}$, Suxian $\mathrm{Ye}^{1,2}$, Jiao Yuan $^{1,2}$ \\ ${ }^{1}$ Guilin University of Technology, Guilin,Guangxi,China \\ ${ }^{2}$ Guangxi Key Laboratory of Spatial Information and Surveying, Guilin,Guangxi,China
}

\begin{abstract}
Virtual Reality has become more and more eye-catching with the development of computer technology in recent years and has become one of the hot technologies. As an important branch of virtual reality, Virtual Campus provides a new way to browse the campus. It uses advanced equipment to simulate the scenes on campus through computer, and gives users a new browsing experience, allowing users to interact with the Internet through the Internet. This article takes the North China Institute of Technology as an example, through SketchUp, Unity3D In a combined way, the development and design of a three-dimensional virtual campus is carried out. A virtual campus system for human-computer interaction in North China Institute of Science and Technology was realized.
\end{abstract}

\section{Introduction}

With the continuous development of computer technology, the performance of hardware equipment has been continuously improved, and the price of equipment has become more and more friendly. Virtual reality technology has developed by leaps and bounds in decades. Virtual reality is not only a technology, but also an interdisciplinary subject of various technologies such as computer graphics, artificial intelligence, sensor technology, and human-computer interaction technology[1]. With the acquisition of Oculus by Facebook, since then, the wave of virtual display products has been rised, and virtual reality has entered a period of rapid development. The rise of virtual reality technology is not only limited to the popular service of entertainment games, but also provides new methods for scientific experiments and simulation. Virtual reality uses computer simulation to generate a virtual world in three-dimensional space, providing users with simulations of visual, auditory, tactile and other senses, allowing users to be as immersive as they can, and can observe the three-dimensional space without limit[2].

Virtual campus is a specific application of virtual reality technology. It utilizes computer virtual reality technology, network technology, strong sense of reality, powerful functions, convenient maintenance and update, etc[3]. It also has intelligent query and positioning function and management background mark function and 3D panorama function. Let users experience a sense of subjective existence and immersion. It is a revolutionary breakthrough in the construction of virtual campus. It can play an important role in display of campus history and cultural, admissions promotion, celebration activities, information inquiry and service, etc. An important method of constructing virtual campus [4]. This article uses SketchUp to build a 3D scene model, Unity3D engine to complete advanced interactive functions such as roaming query, and in-depth discussion of SKETCHUP and Unity3D to build 3D virtual campus optimization technology.

\section{Technical route of virtual campus}

Virtual campus development is divided into two parts: The first is the establishment of a three-dimensional model, in which spatial data is imported into Google SketchUP to build a realistic three-dimensional model.

Spatial data is the basis for building a virtual campus, including topographic maps, building plans, building elevation data, and surface features. The second is to use the $\mathrm{C} \#$ language to code roaming system, query and human-computer interaction functions on the Unity3D platform based on the completion of modeling. The specific process is shown in Figure 1:

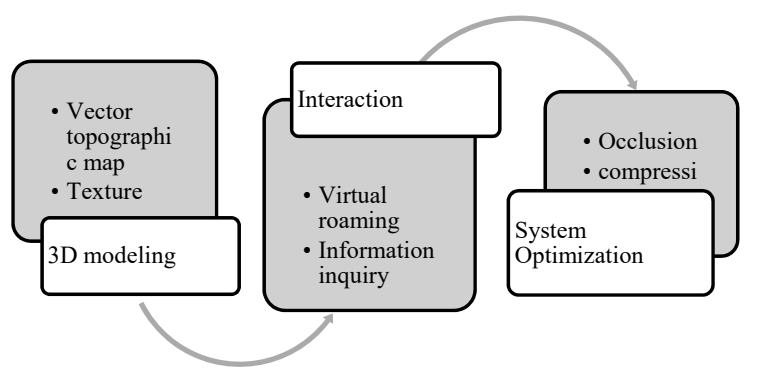

Fig.1. Virtual campus development flow chart

\section{Campus virtual environment construction}

* Corresponding author: huyao@glut.edu.cn 


\subsection{Collection and processing of campus spatial data}

Campus spatial data includes the geographic location of the building, surface texture, spatial relationships, and so on. Therefore, the digital camera is used to collect the surface texture of the building, and the total station uses the non-prism method to measure the elevation information of the building to obtain the spatial data. In addition, the collection of architectural details is required to record geometric information such as the size of each part of the building. Together with the existing topographic map of North China Institute of Technology, you can locate the building and get its outline.

Select the part to be modeled from the topographic map. Since the topographic map is drawn by Cass, there are many unnecessary features for the modeling and also appear on the topographic map. Delete the layer by deleting the layer, delete the unwanted information, and retain the three-dimensional information such as the outline and relative position of the building. Due to the limitation of the viewing conditions, the points on the topographic map and the lines are not all on the same plane, which brings great problems to the modeling. It is necessary to process the topographic map in CAD beforehand, and all the points and lines are placed in the same plane. The entire topographic map has a large range and contains a lot of content. Manual processing is undoubtedly a huge project. It is processed here using the Autolisp language.

\subsection{Establishment of 3D model of campus architecture}

The 3D model not only determines the visual effect that is ultimately presented to the user, but also determines the operational efficiency of the virtual campus[5]. If the model is too fine, the rendering efficiency will be low. Therefore, when modeling, you need to make some choices, you can give up some unimportant buildings and details. Optimize the model by removing invisible faces, selecting the appropriate format, and compressing the map.

Taking library modeling as an example, draw a closed library outline based on existing library topographic maps. Use a digital camera or a total station to perform acquisition-assisted modeling of building appearance data and textures. Use push/pull tools from the bottom up in SketchUp to build a solid model. Then use the arc, zoom and other tools to complete the details. For complex construction components, it can be obtained by Boolean operations such as intersection, union, difference, and construction in SKETCHUP. Then import the acquired texture data to make the model more realistic. Delete redundant information such as invisible surfaces appropriately to reduce the model volume.

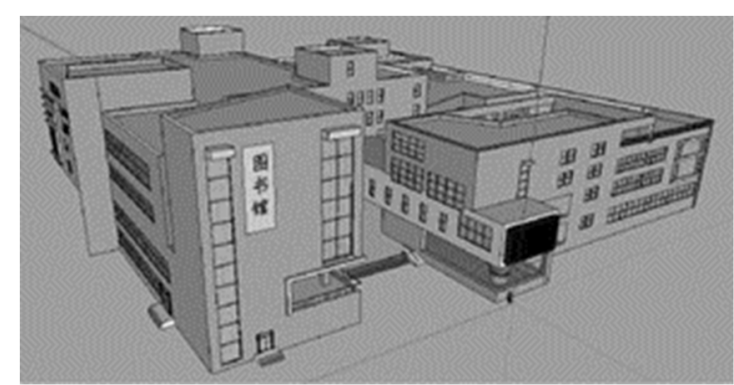

Fig. 2. Library 3D model.

\section{Interactive system construction}

Unity $3 \mathrm{D}$ is a cross-platform $3 \mathrm{D}$ game engine from Unity Technologies that supports the vast majority of desktop and mobile devices. Developed using WYSIWYG development methods, support scripting in C\# language, and code is highly reusable. Unity $3 \mathrm{D}$ is also a large commercial engine.Unity $3 \mathrm{D}$ also has good image quality, with physics-based standard shaders, real-time global illumination, reflection probes and other new technologies, making the rendered image quality more realistic and natural, and better restore the real campus scene. This topic will use Unity3D as the development platform for the virtual campus interactive system.

\subsection{Virtual Roaming}

Virtual roaming is the main function of the main virtual campus. The user observes the virtual environment from the first person perspective.In Unity3D, a character controller with a first-person perspective is provided, which can be added to the scene panel to adjust the appropriate height and field of view for the first perspective.

\subsection{Collision detection}

Collision detection is a simulation of an object in an environment where an obstacle is encountered. For example, when a character encounters a wall, if there is no collision detection, the character will appear through the wall. In this case, the designer needs to add a collision detection function. The conditions required to generate collision information. The collision device in Unity3d is a group of components, which contain many types, such as: Box Collider, Capsule Collider, etc.Objecet has the following interface functions in Unity3d to handle these two collision detections:

Trigger information detection:

1.MonoBehaviour.OnTriggerEnter

2.MonoBehaviour.OnTriggerExit

3.MonoBehaviour.OnTriggerStay

Collision information detection:

1.MonoBehaviour.OnCollisionEnter

2.MonoBehaviour.OnCollisionExit

3.MonoBehaviour.OnCollisionStay 


\subsection{Implementation of information display function}

In the roaming of the virtual environment, the name of each building is displayed to the roamer so that the roamer can be familiar with the virtual environment, and the user can use the function to familiarize with the campus environment. The school can also display relevant information through sub-functions. In passive roaming, producers can easily pass information to a roamer at a specific point in time. However, since roaming is an active roaming, the user needs to actively acquire the information of the building, so a trigger method is needed to display the information. This function is implemented by triggering the information display scheme when hovering with the mouse in this system. In Unity, you need to use the Box Collider to monitor the state of the mouse, and display the prepared information at the specified position when the mouse is hovered.

\subsection{Construction of the sound system}

Sound is also an indispensable part of the virtual environment. Some people talk in the real world, wind, water, etc., and in virtual reality, the sound is divided into two parts: background music and environmental sound. The ambient sound effect is to simulate various sounds that appear in the real world. It has the characteristics that the sound is small from the sound source and the sound is large from the sound source. The sound decay function can be adjusted in Unity to maximize the real sound. Unity's mixing system automatically adjusts the sound size according to the characteristics of the sound when two sounds are superimposed.

\section{5 system optimization}

The optimization of the system is optimized in a very important part after the completion of the system construction, which can improve the system running speed and improve the user experience. If the optimization is not good, the system will be abnormal, and the optimization of the system will mainly refer to the software level. The optimization makes it run smoothly on different configurations computers.

The factors affecting system performance are mainly concentrated in the following aspects:

(1) Excessive vertex-like operations.

(2) Excessive camera-by-camera operations.

(3) The texture resolution is too high.

(4) The script is redundant or complicated.

Since SKETCHUP weakens the concept of normal and triangle, resulting in anomalies in the import of the model, the integrity of the model is crucial. This also causes the same vertex to have the same UV coordinates, and the GPU splits it into multiple vertices when processing, reducing the efficiency of rendering. In this system, the occlusion culling method is mainly used to optimize the scene.
1.Mesh Compression reduces the number of triangular faces of the model when importing models into the Unity editor.

2.After the import of the model is completed, the non-movable objects are set to static. Since the project is a virtual reality project and the scenes are all buildings, the whole model is set to static.

3. Vertical Hold because it is the CRT from the top of the screen to the bottom, and then return to the original position, and its numerical vertical synchronization represents the refresh rate of the CRT display is FPS. If the vertical synchronization is turned off, after the engine finishes rendering an image, the graphics card and the display can start drawing the next screen image without waiting for the vertical synchronization signal, and naturally can fully exert the strength of the graphics card and improve the operation efficiency.

4.In most cases, the object farthest from the camera is rendered first, and the object close to the camera covers the object behind it, so that the far object is rendered twice. The Occlusion Culling technique is a hierarchical view of building a set of potentially visible objects with a virtual camera. Information about visible and invisible objects is calculated. Use this information to determine that only visible objects within the field of view are rendered, while occluded objects are not rendered. This reduces drawing calls and improves system performance.

\section{Conclusion}

This paper takes the Central District of North China Institute of Science and Technology as an example. Based on the development of Unity3D virtual campus roaming system, SketchUp is used as the modeling software. By using the existing geographic information data, the 3D model of the North China Institute of Science and Technology is constructed. Based on this, the Unity3D engine is used to complete the development of virtual roaming, real-time interaction and other functions. After optimization and testing, the realism and real-time of the scene can fully meet the requirements on ordinary multimedia computers.

\section{Foundation items}

1. Development and Application of Intelligent Security Early Warning System for Visitors in Smart Scenic Areas and Time-Space Virtual Reconstruction Technology Item Number: 20170220

2. Research on Intelligent Indoor Positioning Method Based on WIFI and Its Application

Item Number: 2018-B-02

\section{References}

1. Tomasz Mazuryk.Michael Gervautz.Virtual Reality History Applications Technology and Future.Austria.ViennaUniversity of Technology

2. Grigore c. burdea Philippe coiffet.Virtual Reality Technology. second edition.Wiley(2003) 
3. $\mathrm{Lu}$ Z.H. Gu Rong. Cheng Xiangchun. China Education Informationization,(2011)

4. Yan S.F., Luo Y.L., S.L. Design and implementation of digital campus information system based on ArcIMS. Computer Technology and Development,18 (2008).

5. Zhang X.C. Art and Speed of 3D Modeling. 1st Edition. (2010) 